Original Research Paper

\title{
A New Approach to Load Flow Analysis for Radial Distribution System - Application for Medium Voltage Distribution System in Morocco
}

\author{
Saad Ouali and Abdeljabbar Cherkaoui \\ Laboratory of Innovative Technologies (LTI), \\ National School of Applied Sciences, Abdelmalek Essaadi University, Tangier, Morocco
}

Article history

Received: 04-11-2018

Revised: 29-11-2018

Accepted: 13-06-2019

Corresponding Author:

Saad Ouali

Laboratory of Innovative

Technologies (LTI), National

School of Applied Sciences,

Abdelmalek Essaadi

University, Tangier, Morocco

Email: saad.ouali1@gmail.com

\begin{abstract}
This paper presents a load flow algorithm based on the backward/forward sweep principle, flexible for radial distribution system reconfiguration, with an improvement in ensuring a minimum number of searching for connections between nodes in the calculation sequence in the forward and the backward sweep, by organizing the radial distribution system information into a Main-Line and its derivations. The proposed load flow analysis is easy to implement and does not requires the use of any complex renumbering of branches and nodes, or any matrix calculation, with the only use of linear equations based in the Kirchhoff's formulation.
\end{abstract}

Keywords: Load Flow Analysis, Radial Distribution System, Backward/Forward Sweep, Medium Voltage System in Morocco, Advanced Metering Infrastructure

\section{Introduction}

Distribution networks are radial with large number of nodes, branches, distributed generation and complex topology configurations, that can be changed for maintenance activities, emergency operations or network configurations. Due to the high R/X rations, transmission grid load flow methods, or the Jacobienbased methods, such as Newton-Raphson, GaussSeidel and fast-decoupled methods are failed with such networks (Alinjak et al., 2016; Dukpa et al., 2009), even though with some advancements in the NewtonRaphson methods, the robustness of the program is obtained but still the computational time is large (Ouali and Cherkaoui, 2018a; AppaRao and Win Babu, 2013).

These problems have been revealed in a number of papers, where the authors complained that the Jacobienbased methods were not appropriate to solve practical problems presented when analysing distribution systems (Díaz et al., 2015; Shirmohammadi et al., 1988) and prefer Backward Forward sweep methods.

Many studies in the field of Backward Forward Sweep methods improvement and acceleration have been published.

A direct method can be obtained by developing two matrices from the topological structure of the network: Bus Injection to Bus Current (BIBC) and Bus Current to
Bus Voltage (BCBV), the solution is obtained by a simple matrix multiplication of $\mathrm{BIBC}$ and $\mathrm{BCBV}$ (Thakur and Dhiman, 2006; Teng, 2003).

To avoid the use of matrix calculations with large dimension matrices in every iterations of the backward and the forward sweep process, others methods had been developed, with an improvement in ensuring a minimum number of searching for connections between nodes, by renumbering the nodes and branches (Ghosh and Das, 1999; Goswami and Basu, 1991), or by organizing the radial distribution system information into appropriates matrices (Vasquez and Quilumba, 2016), the aim of those improvements is to help the load flow program to begin at the right buses (the terminal bus of each branch) and iterates through the rest of the radial distribution system buses in the correct order.

In this paper, we contribute a new approach of the load flow analysis with a method that does not requires the use of any complex renumbering of branches, or any matrix calculation. The proposed method is an iterative procedure based on Backward/Forward sweep algorithm with the only use of Kirchhoff's formulation. From a dynamic data of system, a preliminary calculation is made to organize the radial distribution information into a Main-Line and its Derivations. After the organization of the system data information into a suitable model. Voltages of all other nodes are set to the nominal 
voltage and an iterative process start by calculating currents of branches in a backward sweep using the Kirchhoff's current law and the node voltages are updating in a forward sweep using the voltage drop calculation, this backward and forward sweep are repeated until voltage magnitudes at each node in present iteration and previous iteration is lower than a tolerance limit. An IEEE 15-bus radial distribution system is taken as a study system for performing the test of the proposed method, the results shown are compared with three existing load flow methods presented in (Ouali and Cherkaoui, 2018b): Primitive Impedance based Distribution Load Flow (PIDLF), Current Injections based Distribution Load Flow (CIM) and Fast Decoupled Single Matrix Model Distribution Load Flow (SMM).

The main contributions of this paper is presenting a simple load flow method to implement, where the complex branch renumbering is not needed and it's independent from the first numbering of nodes, the reducing flow calculation is obtained by ensuring a minimum number of searching for connections between nodes and by using only simple algebraic expressions without any trigonometric functions or matrix calculation. The proposed load flow method is valid for radial distribution system with or without distributed generations and it's flexible for the network reconfiguration.

In what follows, we present an overview of the medium voltage distribution system in Morocco in section II. Next in Section III we provide the theoretical foundation and the algorithm, In section IV we discuss the results obtained from a 15-bus test system and the comparison of the results with other existing load flow methods. Section V concludes this paper.

\section{Moroccan Medium Voltage Distribution System}

The Moroccan medium voltage distribution system is made of 3 phases, with neutral grounded in the HV/MV substation. Two nominal magnitudes voltages are used $20 \mathrm{KV}$ and $22 \mathrm{KV}$.

Three forms of medium voltage distribution system can be distinguished: 100\% overhead lines, 100\% underground cables or a combination of both.

The underground networks are made of 3 singlephase cables. The most used topology is the open loop (ring configuration) in which each MV/LV substation is connected to at least the two others substations, as shown on Fig. 1, with rare cases of MV/LV substation in antenna.

The single line diagram of the open-loop topology is shown on Fig. 2.

Several structures exist in Morocco, a very simple complete structure can be obtained by having feeders from $\mathrm{HV} / \mathrm{MV}$ substation to another HV/MV substation, as shown in Fig. 3.

The overhead networks are made of three bares conductors, the most used topology is the radial configuration, as shown on Fig. 4, it's to highlight that is recommended to avoid the use of the rake structure (a connection from the main line for each station), which weakens the main-line and it's preferable to grouping as many station, into cluster, as possible. (Minimize the number of derivations).

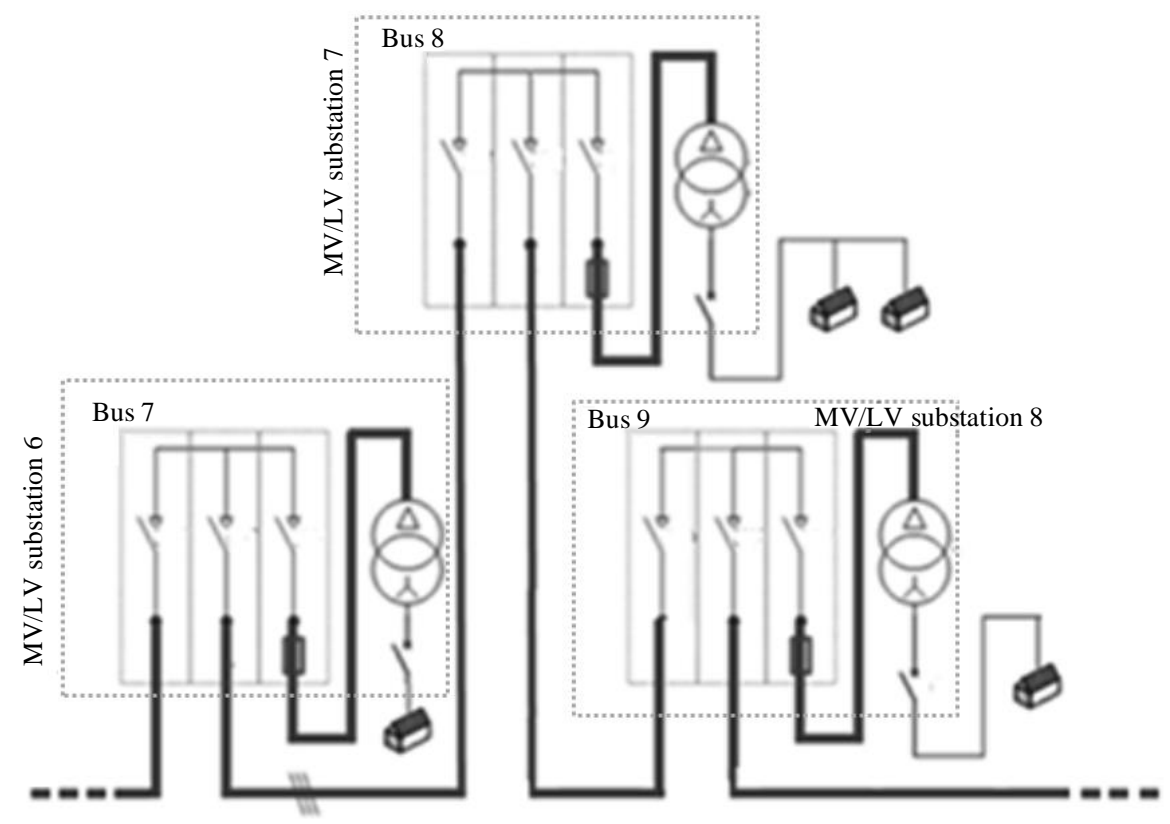

Fig. 1: Open-loop topology 


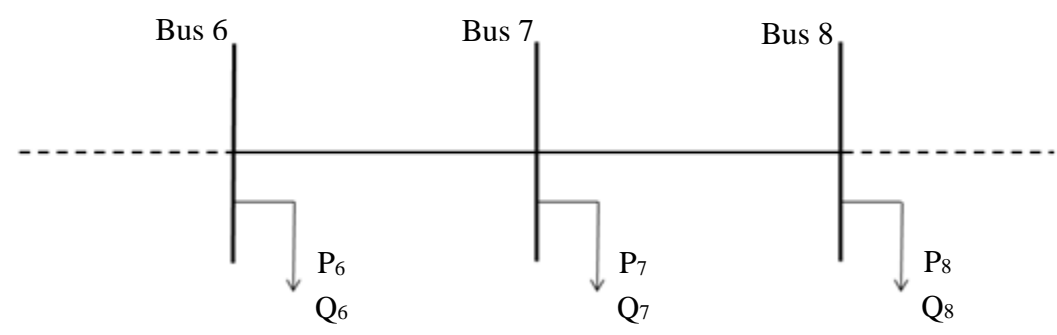

Fig. 2: Single line diagram of the open-loop topology

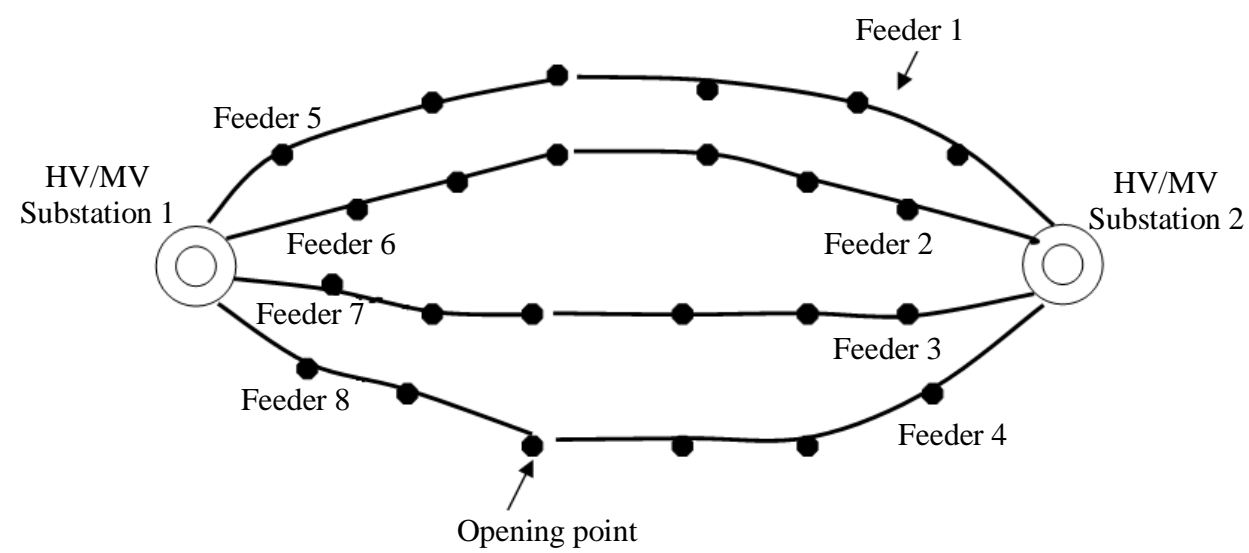

Fig. 3: A complete structure of the open-loop topology with feeders from HV/MV substation to another HV/MV substation

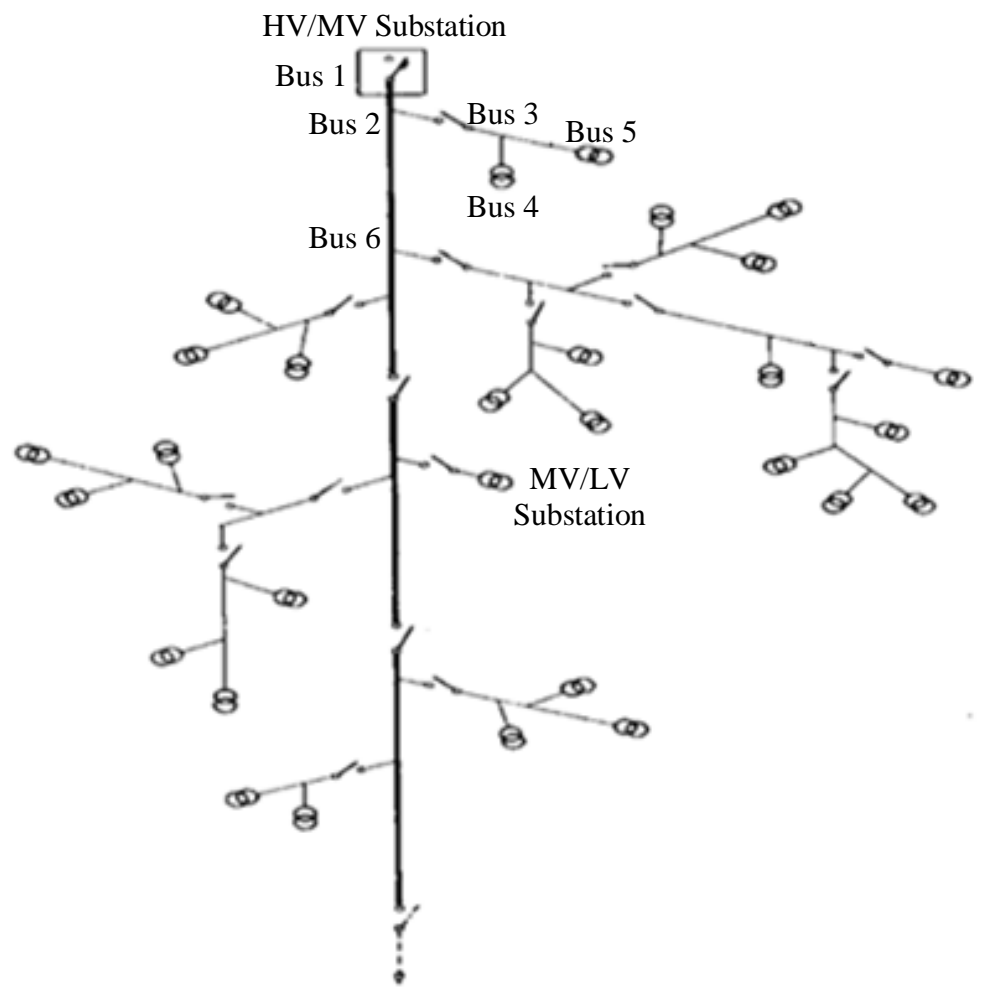

Fig. 4: Radial configuration of overhead distribution system 
A complete structure is obtained by looping-back feeders in way to save a large part of the feeder in case of incidents or maintenance activities.

According to these several forms of the Medium voltage, it's possible to separate three types of nodes, as presented in Fig. 5: Terminal node, common node and intermediate node.

\section{Algorithm}

From a dynamic Line-Data of the system, a preliminary calculation is made to organize the radial distribution information into a suitable model, the proposed element ordering process is carried out to determine the calculation sequence in the forward and the backward sweep, by dividing the network into a "Main-Line" and its "derivations".

The Main-Line, in this paper, is chosen as the longest sequence of nodes. It's to highlight that the Main-Line can be chosen according to the need: The most loaded line or the line allowing a backup in the case of the open loop structure.

From the Common buses of the main-line, derivations are obtained and organized into an appropriate matrix $\mathrm{D}$, the form of the matrix D is shown in Fig. 6.

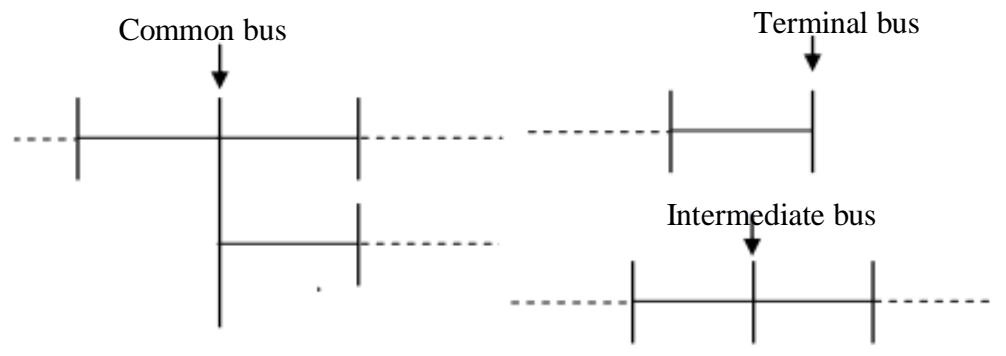

Fig. 5: Types of nodes

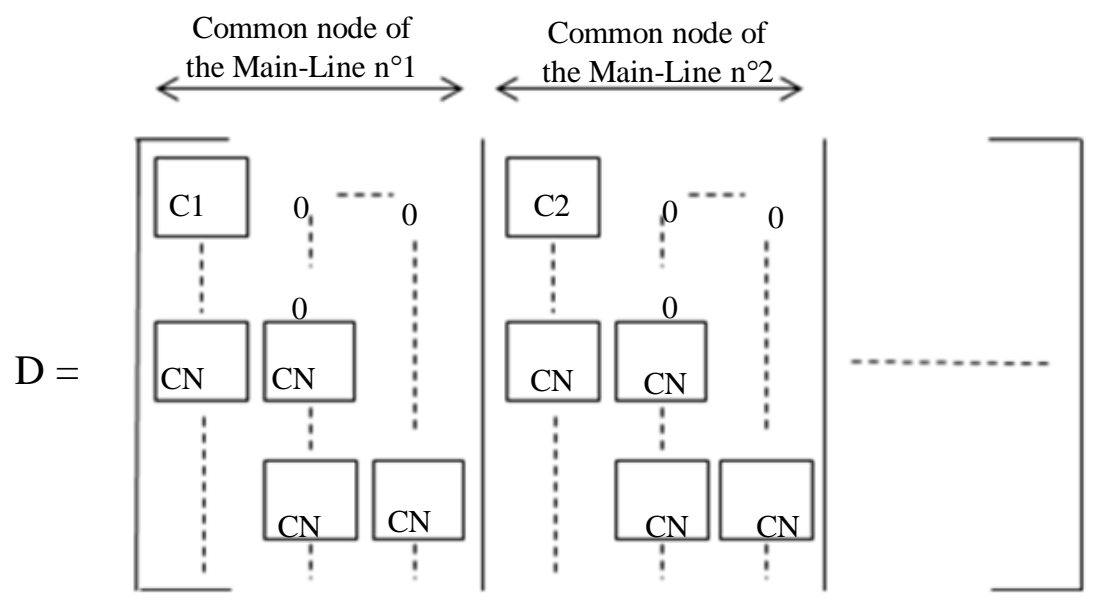

Fig. 6: Derivation matrix

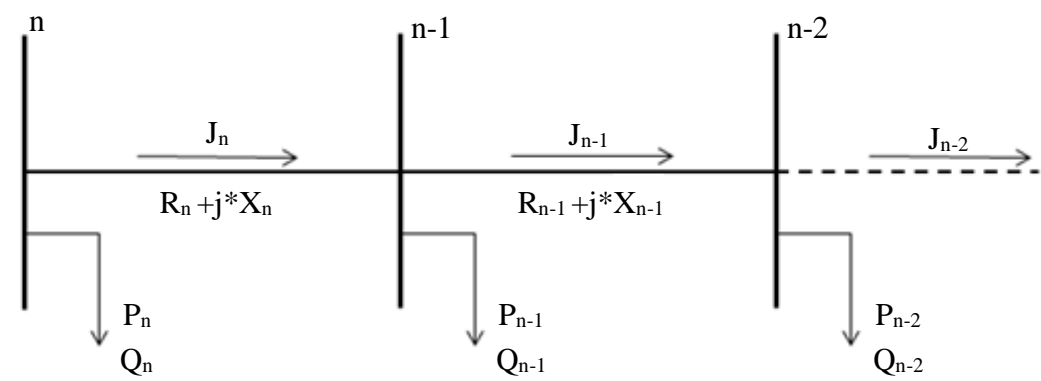

Fig. 7: Single line diagram 
With $\mathrm{C} 1$ is the first common node of the main-line and $\mathrm{C} 2$ the second common node. A table of the common buses of the main-line is made to facilitate the programming of the proposed algorithm, this table contains 5 columns: The common nodes of the mainline, the next node in the main-line, number of nodes linked to the common bus, the number of derivation stemming from this common node and the sum of branch currents of its derivation.

After the organization of the Line Data information, the next step is to read the Load-Data and convert it into a per unit values and set the voltages of all nodes to the nominal voltage "1p.u".

By preparing the Line Data and the Load Data information into a suitable model, an iterative procedure based on Backward/Forward sweep algorithms start.

The backward sweep: This step is based on the fact that the complex power is known at each node. Starting from the terminal node of each derivation:

The electric current between the terminal node " $n$ " and the next node " $n-1$ ", shown in Fig. 7, is:

$$
J_{n}^{k}=\operatorname{conj}\left(\frac{P_{n}+j^{*} Q_{n}}{V_{n}^{k}}\right)
$$

The electric current between a sending node $(n-i)$ and a receiving node $(n-i-1)$ is:
$J_{n-i}^{k}=-\operatorname{conj}\left(\frac{P_{n-i}+j^{*} Q_{n-i}}{V_{n-i}^{k}}\right)+\sum_{r} J_{n-r}^{k} r=1 \ldots$

where, $\sum_{r} J_{n-r}^{k}$ is the currents in branches emanating from the node "n-i".

The forward Sweep: Using the computed current, an updating of voltages values start from the root bus, the node voltages are updated using the Equation (3):

$$
V_{n}^{k}=V_{n-1}^{k}-\left(R_{n}+j * X_{n}\right) * J_{n}^{k}
$$

This backward and forward sweep is repeated until voltage magnitudes at each node in present iteration and previous iteration is lower than a tolerance limit.

The flow chart of the proposed method is given in Fig. 9.

\section{Simulation Results}

The proposed method program code is made in MATLBAB and tested on IEEE 15-bus distribution network, the Line diagram of this network is shown in Fig. 8. The IEEE 15-bus presents similar structure as Moroccan medium voltage distribution system, the Line-Data is given in Table 1 and a Load-data is presented in Table 2, the power factor of load is assumed to be 0.7 (Tripathy and Singh, 2004).

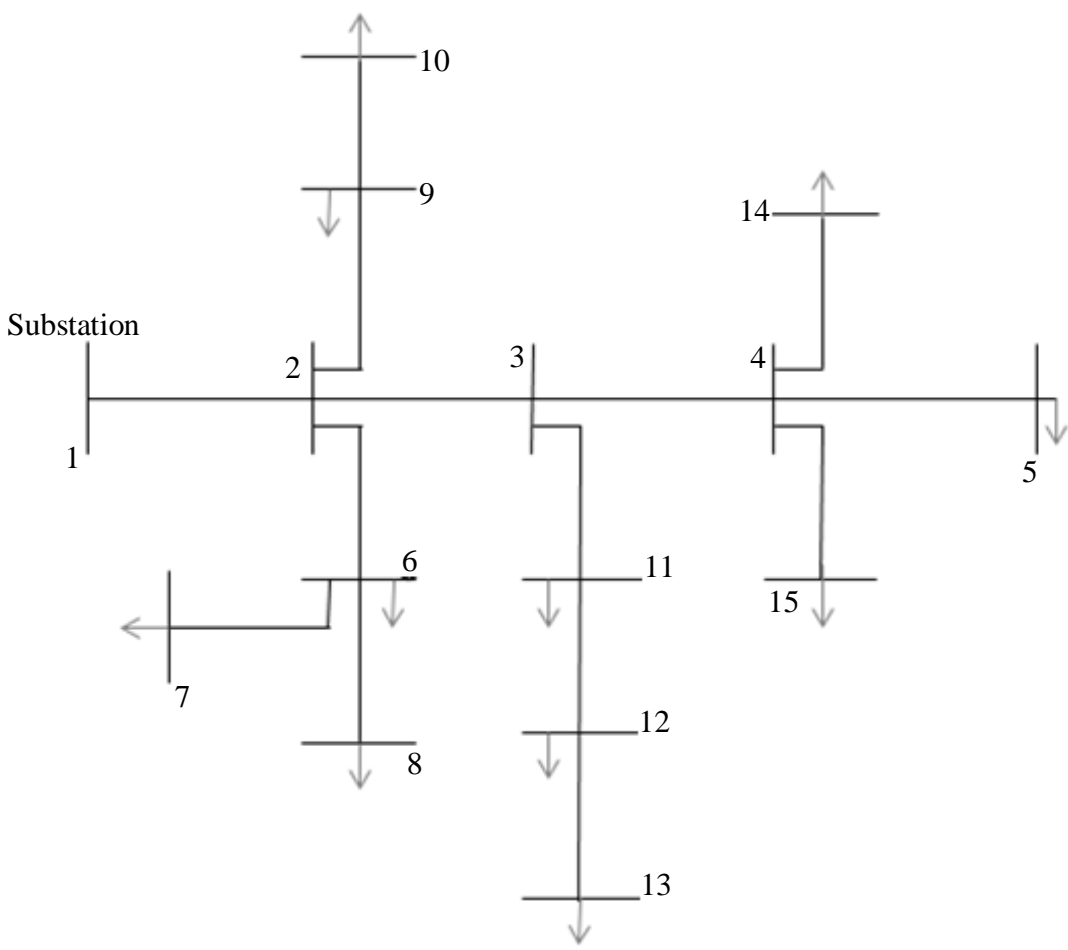

Fig. 8: Single phase diagram of 15-bus distribution system 


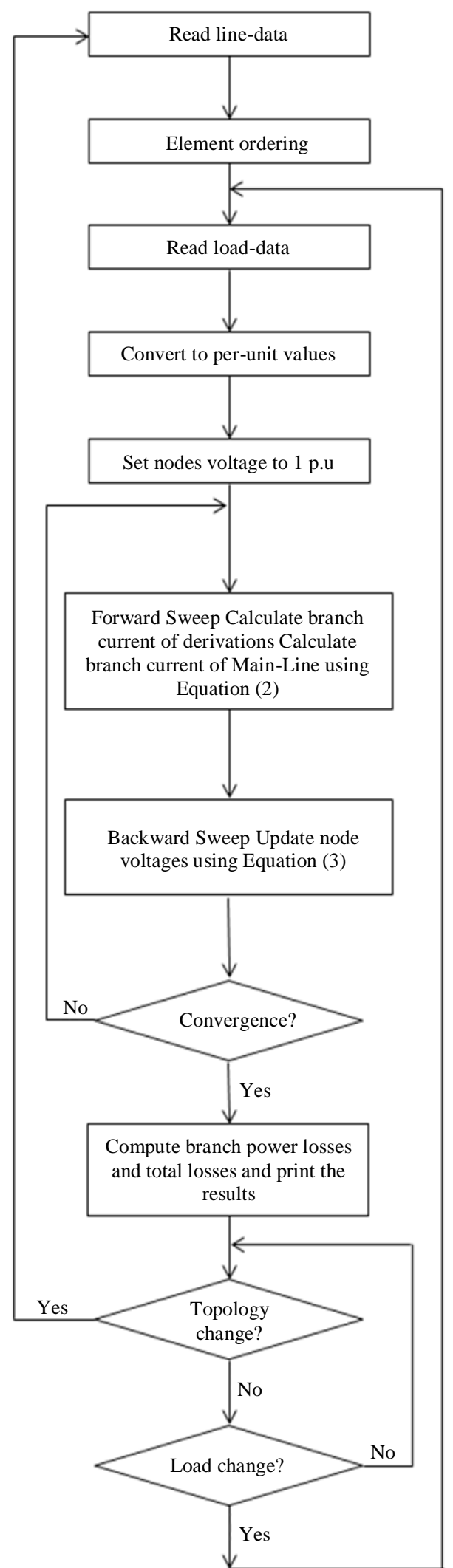

Fig. 9: Flow chart of the proposed method

$$
D=\left[\begin{array}{ccc|c|cc}
\stackrel{\text { Derivation1 }}{3} & 0 & 0 & 2 & \multicolumn{2}{c}{\text { D2 }} \\
4 & 4 & 4 & 9 & 6 & 6 \\
4 & 14 & 15 & 10 & 7 & 8
\end{array}\right]
$$

Fig. 10: The matrix of derivations

$$
c b m l=\left[\begin{array}{ccccc}
3 & 11 & 2 & 3 & 1.7617-4.8878 i \\
2 & 3 & 3 & 6 & 2.6069-2.6565 i
\end{array}\right]
$$

Fig. 11: Matrix of the common buses of the Main-Line

Table 1: Load data of the 15-bus system

\begin{tabular}{lccccc}
\hline Node & KVA & Node & KVA & Node & KVA \\
\hline 1 & 00.0 & 6 & 200.0 & 11 & 200.0 \\
2 & 63.0 & 7 & 200.0 & 12 & 20.0 \\
3 & 100.0 & 8 & 100.0 & 13 & 100.0 \\
4 & 200.0 & 9 & 100.0 & 14 & 100.0 \\
5 & 63.0 & 10 & 63.0 & 15 & 200.0 \\
\hline
\end{tabular}

Table 2: Line data of the 15-bus system

\begin{tabular}{lcll}
\hline Sending node & Receiving node & $\mathrm{R}(\mathrm{Ohm})$ & $\mathrm{X}(\mathrm{Ohm})$ \\
\hline 1 & 2 & 1.530 & 1.778 \\
2 & 3 & 1.037 & 1.071 \\
3 & 4 & 1.224 & 1.428 \\
4 & 5 & 1.262 & 1.499 \\
2 & 9 & 1.176 & 1.335 \\
9 & 10 & 1.100 & 1.190 \\
2 & 6 & 1.174 & 1.332 \\
6 & 7 & 1.174 & 1.332 \\
6 & 8 & 1.174 & 1.323 \\
3 & 11 & 1.150 & 1.285 \\
11 & 12 & 1.274 & 1.522 \\
12 & 13 & 1.274 & 1.522 \\
4 & 14 & 1.075 & 1.522 \\
4 & 15 & 1.075 & 1.522 \\
\hline
\end{tabular}

From the dynamic Line-Data, presented on Table 1, a preliminary calculation is made to organize the radial distribution information into a suitable model: A "MainLine" and its "derivations".

The Main-Line, the line containing the largest number of successive nodes, of the 15-Bus system is [1 2 $\left.\begin{array}{llll}3 & 11 & 12 & 13\end{array}\right]$, with 6 nodes. (as shown in red in the Fig. 11 $\mathrm{N}^{\circ} 12$ ) and the three derivations have been identified from the common nodes 2 and 3 .

The matrix D is shown on Fig. 10.

And the matrix of common buses of the main-line is.

Matrix of common buses of the main-line helps to browse all elements of the matrix of derivation " $D$ ", by identifying the columns belonging to each derivation.

The element ordering of the IEEE 15-Bus is presented in Fig. 12.

After the organization of the Line-Data information into a Main-Line and its derivations, the next step is to read the Load- Data, presented in Table 2 and convert it into a per unit values and set all the voltage value of all nodes to "1 p.u". 


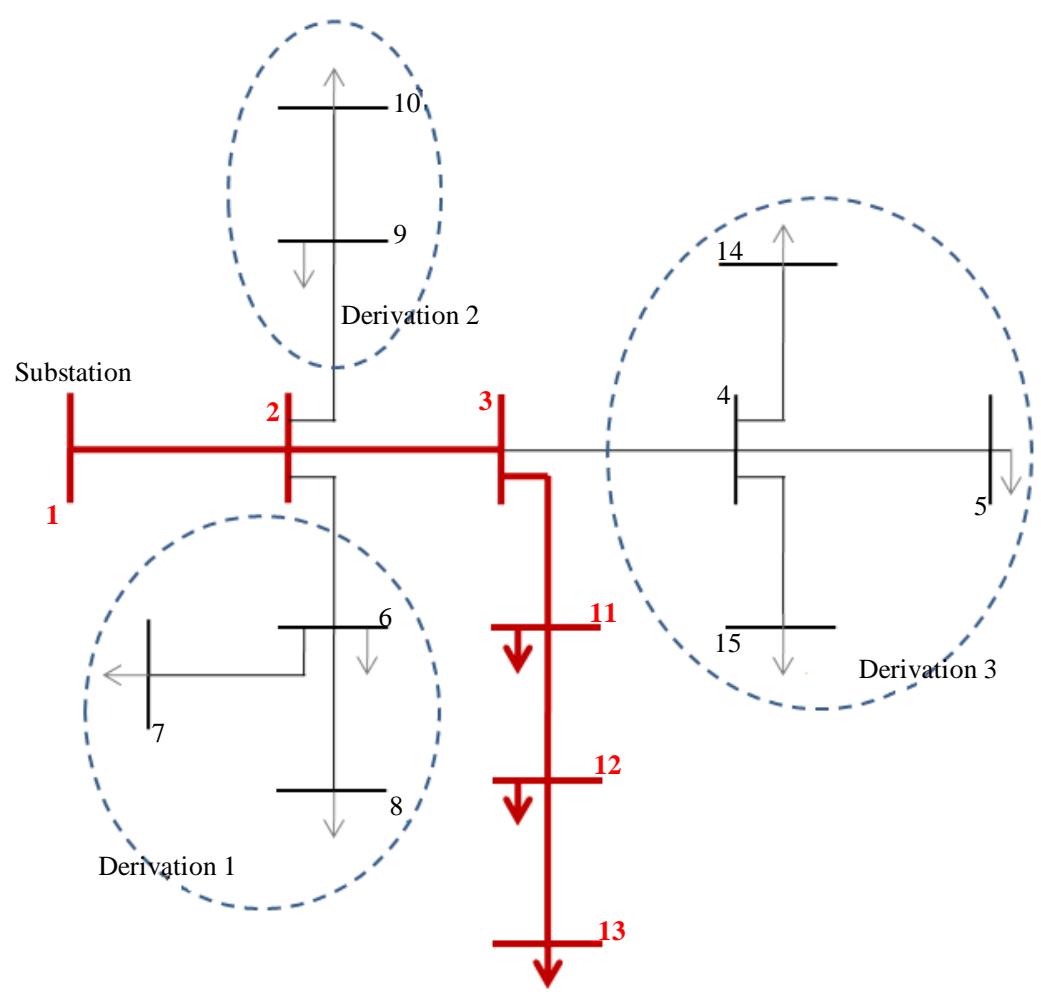

Fig. 12: The Main-Line and its derivation of the 15-bus system

Table 3: Results for IEEE 15-bus system

\begin{tabular}{|c|c|c|c|c|}
\hline Node & CIM & PIDLF & SMM & Proposed method \\
\hline$\overline{1}$ & 1.00000 & 1.00000 & 1.00000 & 1.00000 \\
\hline 2 & 0.97128 & 0.96885 & 0.97031 & 0.97017 \\
\hline 3 & 0.95667 & 0.95427 & 0.95571 & 0.95657 \\
\hline 4 & 0.95090 & 0.94852 & 0.94995 & 0.95078 \\
\hline 5 & 0.94991 & 0.94754 & 0.94896 & 0.94977 \\
\hline 6 & 0.95822 & 0.95583 & 0.95726 & 0.96267 \\
\hline 7 & 0.95476 & 0.95237 & 0.95380 & 0.95974 \\
\hline 8 & 0.95694 & 0.95455 & 0.95599 & 0.96115 \\
\hline 9 & 0.96797 & 0.96555 & 0.96700 & 0.96020 \\
\hline 10 & 0.96689 & 0.96448 & 0.96593 & 0.95936 \\
\hline 11 & 0.94995 & 0.94757 & 0.94900 & 0.95202 \\
\hline 12 & 0.94582 & 0.94346 & 0.94488 & 0.95006 \\
\hline 13 & 0.94451 & 0.94215 & 0.94357 & 0.94842 \\
\hline 14 & 0.94860 & 0.94623 & 0.94766 & 0.94926 \\
\hline 15 & 0.94844 & 0.94606 & 0.94749 & 0.94775 \\
\hline
\end{tabular}

Base voltage and base power are considered as $11 \mathrm{KV}$ and 100KVA, respectively.

By preparing the Line Data and the Load Data information into a suitable model, an iterative procedure based on Backward/Forward sweep algorithms start, the convergent criteria is chosen as 0.0001 .

The results obtained was been compared with three others existing load flow methods (Ouali and Cherkaoui, 2018): Primitive Impedance based Distribution Load Flow (PIDLF), Current Injections based Distribution Load Flow (CIM) and Fast Decoupled Single Matrix
Model Distribution Load Flow (SMM). The results are summarized on Table 3.

\section{Conclusion}

In this new approach, a load flow program based on the backward/forward sweep concept, is used to solve load flow problems in radial distribution systems, with an improvement in the element ordering that does not requires the use of any complex numbering of branches or the use of any matrix calculation, simply by devising the 
system elements into a Main-Line and its derivations, the flow calculation is reduced due to the use of only algebraic expressions without any trigonometric functions or matrix calculation and by ensuring minimum a number of searching for connections between nodes.

The proposed Load flow method is easy to implement in the current Moroccan medium voltage system, a Load-Data can be obtained and updated by the Advanced Metering Infrastructure (AMI), already existing and used for consumers connected to the medium voltage distribution network in Morocco, the needed Data of others loads can be obtained by equipping $\mathrm{MV} / \mathrm{LV}$ substation by digital meters and connect them to the Advanced Metering Infrastructure network. The Line-Data can be established by collecting the impedance of all the branches constituting the network, any change in the topology structure can be detected manually or by the existing SCADA, installed already in HV/MV substation in Morocco and used as medium voltage network remote control system.

The proposed method can be exploited in several distribution network applications: Voltage control, demand side management, energy demand management.

Limitation of the proposed method is that it can be used only for the radial distribution system and, not for meshed distribution systems or transmission systems.

\section{Author's Contributions}

Saad Ouali and Abdeljebbar Cheraoui: Performed the approach development, the simulation work and conducted analysis of the research. All authors contributed to the writing of the manuscript.

\section{Ethics}

The authors declare that there are no ethical issues that could arise after the publication of this study.

\section{References}

Alinjak, T., I. Pavić and M. Stojkov, 2016. An improvement of Backward/forward sweep power flow method by using modified Breadth-first search strategy. IET Generat. Transmiss. Distribut.

AppaRao, A. and M. Win Babu, 2013. Forward sweeping method for solving radial distribution networks. IJAREEIE, 2: 4305-4311.
Díaz, G., J.G. Aleixandre and J. Coto, 2015. Direct backward/forward sweep algorithm for solving load power flows in AC droop-regulated microgrids. IEEE Trans. Smart Grid, 7: 2208-2217. DOI: 10.1109/TSG.2015.2478278

Dukpa, A., B. Venkatesh and M. El-Hawary, 2009. Application of continuation power flow method in radial distribution systems. Elect. Power Syst. Res., 79: 1503-1510. DOI: 10.1016/j.epsr.2009.05.003

Ghosh, S. and D. Das, 1999. Method for load-flow solution of radial distribution networks. IEE Proc. Gener. Transm. Distr., 146: 641-48. DOI: 10.1049/ip-gtd:19990464

Goswami, S.K. and S.K. Basu, 1991. Direct solution of distribution system. IEE Proc. C, 188: 78-88. DOI: $10.1049 /$ ip-c. 1991.0010

Ouali, S. and A. Cherkaoui, 2018a. Load flow analysis for moroccan medium voltage distribution system. Proceedings of the Engineering and Technology Conférence Internationale en Automatique and Traitement de Signal, (ATS’ 18) pp: 10-16.

Ouali, S. and A. Cherkaoui, 2018b. Sensitivity analysis in Medium voltage distribution systems. Int. J. Eng. Res. Technol., 7: 43-49.

Shirmohammadi, D., H. Hong, A. Semlyen and G. Luo, 1988. A compensation-based power flow method for weakly meshed distribution and transmission networks. IEEE Trans. Power Syst., 3: 753-762. DOI: $10.1109 / 59.192930032$

Teng, J.H., 2003. A direct approach for distribution system load flow solutions. IEEE Trans. Power Delivery, 18: 882-887.

DOI: 10.1109/TPWRD.2003.813818

Thakur, T. and J. Dhiman, 2006. A new approach to load flow solutions for radial distribution system. Proceedings of the IEEE/PES Transmission and Distribution Conference and Exposition: Latin America, Aug. 15-18, IEEE Xplore Press, Caracas, Venezuela, pp: 1-6. DOI: $10.1109 /$ TDCLA.2006.311634

Tripathy, P. and S.N. Singh, 2004. Power flow analysis with optimally placed d-STATCOM in distribution system. Proceedings of the National Power Systems Conference, (PSC' 04).

Vasquez, W.A. and F.L. Quilumba, 2016. Load flow method for radial distribution systems with distributed generation using a dynamic data matrix. Proceedings of the IEEE Ecuador Technical Chapters Meeting, Oct. 12-14, IEEE Xplore Press, Guayaquil, Ecuador, pp: 1-5. DOI: 10.1109/ETCM.2016.7750862 